\title{
Actividad antibacteriana de extractos de hongos aislados de raíces del mangle Rhizophora mangle (Rhizophoraceae) en Venezuela
}

\author{
Isabel Castillo-Machalskis ${ }^{1 *}$, Haydelba D’ $\operatorname{Armas}^{2}$, Nora Malaver $^{3} \&$ Maximiano Núñez ${ }^{4}$ \\ 1 Instituto Oceanográfico de Venezuela, Universidad de Oriente (UDO-Sucre), Av. Universidad, Cerro Colorado, \\ Código postal 6101, Cumaná, Venezuela. \\ 2 Universidad de Oriente, Núcleo de Sucre, Escuela de Ciencias, Departamento de Química, Venezuela. \\ 3 Universidad Central de Venezuela, Instituto de Zoología Tropical, Laboratorio de Microbiología Ambiental. \\ 4 Universidad de Oriente, Núcleo de Sucre, Instituto Oceanográfico de Venezuela, Departamento de Biología pesquera; \\ maxpaulinon@cantv.net
}

Recibido 12-X-2005. Corregido 27-X-2006. Aceptado 08-V-2007.

\begin{abstract}
Anti-bacterial activity of extracts from fungi collected from mangrove Rhizophora mangle (Rhizophoraceae) roots in Venezuela. The antibacterial activity of marine fungi extracts was evaluated by a test of efficiency. The fungi were previously inoculated in Malt Agar (EMA) extract with $50 \%$ of seawater and growth for 60 days. Triplicate antibiograms were carried out with the extracts. An ANOVA I with a posteriori Duncan test were applied to the diameters of inhibition zones. The extracts of Aspergillus ochraceus 3MCMC3 and Penicillium citrinum (14) 4MCMC16 present wide spectral antibacterial properties, inhibiting $100 \%$ and $80 \%$ of the developing germs, especially Pseudomonas aeruginosa 9027 . The extract of Penicillium (1) 3MLLC5 had the highest efficiency on this strain; P. aeruginosa 9027 and Escherichia coli 10536 were the most sensitive germs when treated with these extracts. These marine fungi can be an important source of antibacterial secondary metabolites. Rev. Biol. Trop. 55 (3-4): 761-765. Epub 2007 December, 28.
\end{abstract}

Key words: antibacterial activity, marine fungi, mangrove roots, Rhizophora mangrove, Tacarigua Lagoon, Venezuela.

La búsqueda de metabolitos provenientes de los microorganismos marinos, representa un campo de rápido crecimiento, y esto es debido principalmente a la sospecha de que algunos de los metabolitos obtenidos de macroalgas e invertebrados pueden ser producidos por los microorganismos epibiontes (Kelekom 2002).

De los trabajos de investigación referidos al aislamiento de hongos marinos con propiedades antibacterianas pertenecientes a costas venezolanas se pueden mencionar los realizados por Christophersen et al. (1999), quienes obtuvieron extractos de 227 hongos marinos bioactivos; Nielsen et al. (1999) obtuvieron el depsido guisinol con actividad antibacteriana a partir del hongo Emericela unguis; Malstrom et al. (2000) mostraron que se establecen patrones de similitud entre los metabolitos secundarios producidos por diferentes especies pertenecientes al género Penicillium, lo cual complementa su identificación. Entre otros trabajos de investigación que reportan metabolitos secundarios bioactivos obtenidos de hongos marinos se encuentran los realizados por Sperry et al. (1998) quienes caracterizaron un policétido al que denominaron epoxysorbicilinol obtenidos del hongo Tricoderma longibrachiatum aislado de la esponja Haliclona sp.; Jenkins et al. (1998) encontraron tres nuevos alfa-pironas, con actividad antimicroalgal provenientes de hongos marinos filamentosos aislados del alga verde Halimeda moline, Hoeller et al (1999), detectaron actividad antimicrobiana en los hongos marinos Coniothyrium sp. y Microphaeropsis 
sp. aislados de la esponja marina Ectyplasia perox y del crustáceo Myxilla sp., respectivamente. En este sentido, los hongos marinos se han mostrado como fuentes de metabolitos secundarios novedosos con cualidades antibacterianas (Jenkins et al. 1998, Sperry et al. 1998, Christophersen et al. 1999, Hoeller et al. 1999, Nielsen et al. 1999, Malstrom et al. 2000, Lin et al. 2002, Shigemori et al. 2004).

El presente trabajo de investigación se basa en la aplicación de una prueba de eficiencias antibacteriana a extractos de hongos marinos. Estos resultados permitieron establecer criterios para escoger la(s) cepa(s) que presente(n) características antibacterianas idóneas, para continuar estudios posteriores dirigidos al aislamiento de sustancias bioactivas con aplicabilidad farmacológica.

\section{MATERIALES Y MÉTODOS}

Procedencia de las muestras: los hongos marinos fueron suministrados por el laboratorio de Microbiología Ambiental del Instituto de Zoología Tropical (IZT) de la Universidad Central de Venezuela (UCV), los cuales fueron aislados de raíces del mangle Rhizophora mangle de la Laguna de Tacarigua, Edo. Miranda (entre los $10^{\circ} 18^{\prime} 00^{\prime} \mathrm{N}, 65^{\circ} 55^{\prime} 01^{\prime \prime} \mathrm{W}$ y $\left.10^{\circ} 12^{\prime} 05^{\prime} \mathrm{N}, 65^{\circ} 43^{\prime} 00^{\prime \prime} \mathrm{W}\right)$, e identificados con la siguiente nomenclatura: Penicillium sp. (1) 3MLLC5, Penicillium citrinum (14) 4MCMC16, Penicillium citrinum (1) 4MCMC4, Aspergillus niger (1) 4MCMC8, Aspergillus ochraceus $3 \mathrm{MCMC} 3$, Trichoderma viride (1) 4MCMC1, Syncephalastrum racemosum (2) 4MCMC10.

Elaboración de los cultivos y obtención de los extractos: para realizar las extracciones, los hongos fueron inoculados en $100 \mathrm{ml} \mathrm{de}$ Agar Extracto Malta (EMA) con $50 \%$ de agua de mar filtrada y envejecida, en matraces de 1 $\mathrm{L}$ en forma inclinada; transcurrido un tiempo de incubación de 60 días a $25^{\circ} \mathrm{C}$, se procedió a llevar a cabo la obtención de los extractos según la metodología expuesta por Christophersen et al. (1999), empleando para la extracción una solución de acetona/cloroformo/etanol (3:2:1).

Actividad antibacteriana: la prueba antibacteriana se llevó a cabo según el método de antibiograma descrito por Bauer et al. (1966), empleando discos de papel Whatman $\mathrm{N}^{\circ} 3$ de $10 \mathrm{~mm}$ de diámetro impregnados con $25 \mu \mathrm{l}$ de una solución de concentración de $40 \mathrm{mg} / \mathrm{ml}$ de los extractos a probar. Se utilizaron como gérmenes reveladores las cepas ATCC Gram negativas: Escherichia coli 10536, Pseudomonas aeruginosa 9027, Salmonella typhimurium 14028; y las cepas Gram positivas: Bacillus cereus 9634 y Staphylococcus aureus 25923. Los antibiogramas se elaboraron por triplicado con sus respectivos controles, con un período de preincubación de $12 \mathrm{~h}$ a $4{ }^{\circ} \mathrm{C}$ e incubación de 24 a $48 \mathrm{~h}$ a $35^{\circ} \mathrm{C}$; los halos de inhibición obtenidos se midieron con ayuda de un vernier digital (Mitutoyo; $0.01 \mathrm{~mm}$ de apreciación).

Eficiencia de los extractos: la eficiencia de los extractos se determinó mediante comparación de los halos de inhibición de los extractos sobre cada microorganismo revelador, aplicando Análisis de Varianza de una vía. A los resultados que presentaron diferencias significativas, se les realizó la prueba a posteriori Duncan (Zar 1984).

\section{RESULTADOS}

Se encontró que el extracto de $A$. ochraceus 3MCMC3, exhibió el mayor espectro de acción inhibiendo el $100 \%$ de los gérmenes reveladores, sin embargo, se evidenció una eficiencia antibacteriana similar sobre las bacterias en estudio. En este sentido, el extracto de P. citrinum (14) 4MCMC16 inhibió el $80 \%$ de los gérmenes reveladores, resultando ser más eficiente sobre E. coli 10536 , con promedio de halos de inhibición de $17.32 \mathrm{~mm}$, el resto de las cepas bacterianas fueron inhibidas en forma similar (ANOVA I $\alpha=0.01 ; \mathrm{F}=511.19$; Duncan $\alpha=0.01$ ) (Cuadro 1, Fig. 1). 
HONGOS MARINOS

Penicillium sp. (1) 3MLLC5

Penicillium citrinum (14) 4MCMC16

Penicillium citrinum (1) 4MCMC4

Aspergillus niger (1) 4MCMC8

Aspergillus ochraceus 3MCMC3

Sincephalastrum racemosum (2) 4MCMC10

Trichoderma viride (1) 4MCMC1

Porcentaje de inhibición de los extractos de hongos marinos $(\%)$

\section{BACTERIAS}
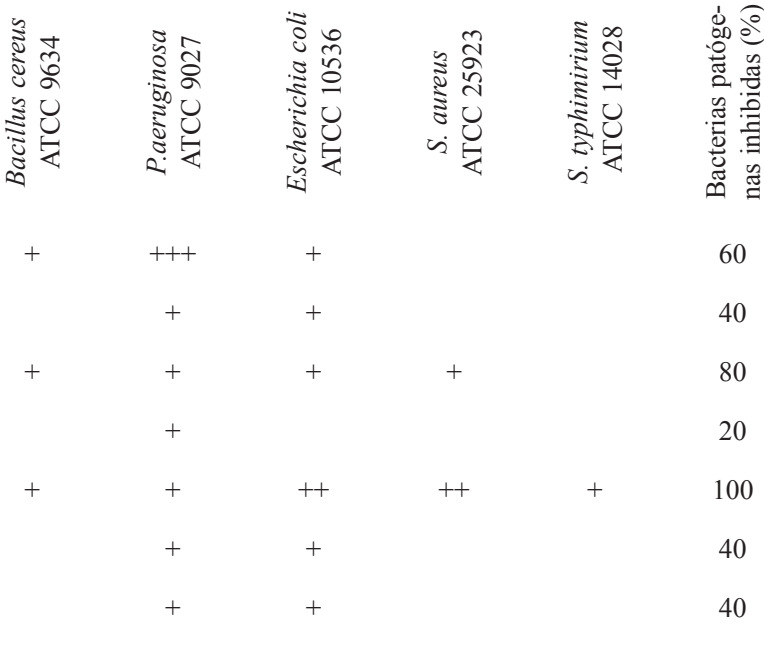

60

40

80

20

100

40

40

$\begin{array}{lllll}42.86 & 100 & 85.71 & 28.57 & 14.29\end{array}$

Valores de halos de inhibición: 11.00-22.00 mm (+); 23.00-30.00 mm (++); 31.00-35.00 mm (+++).

Por otra parte se determinó que $P$. aeruginosa 9027 y E. coli 10536 resultaron ser inhibidos por el $100 \%$ (7) y $85.71 \%$ (6) de los extractos ensayados, presentando el mismo grado de efectividad antibacteriana sobre $E$. coli 10536; sin embargo, el extracto obtenido de Penicillium sp. (1) 3MLLC5 resultó ser el más eficiente sobre $P$. aeruginosa 9027, con promedio de los halos de inhibición de 33.56 mm; mientras que los extractos de $A$. ochraceus 3MCMC3 y P. citrinum (1) 4MCMC4 resultaron ser los menos eficientes sobre este germen revelador, con promedios de los halos de inhibición de $13.4 \mathrm{~mm}$ y $14.86 \mathrm{~mm}$ ANOVA I $(\alpha=0.01 ; F=31.49)$; Duncan $(\alpha=0.01)$. (Cuadro 1, Fig. 2).

\section{DISCUSIÓN}

Los resultados obtenidos en el presente estudio no concuerdan totalmente con los encontrados por Christophersen et al. (1999), quienes encontraron cepas de hongos marinos pertenecientes a los géneros Penicillium o Aspergillus, las cuales inhibieron solo el crecimiento de $S$. aureus 25923 o Vibrio parahaemolyticus 17802, también observaron que estos hongos no inhibieron el crecimiento de Escherichia coli 25922 y P. aeruginosa 27853. De manera similar, Nielsen et al. (1999) encontraron que el metabolito Guisinol aislado del hongo marino Emericella unguis inhibió el crecimiento de una cepa de $S$. aureus, a su vez 


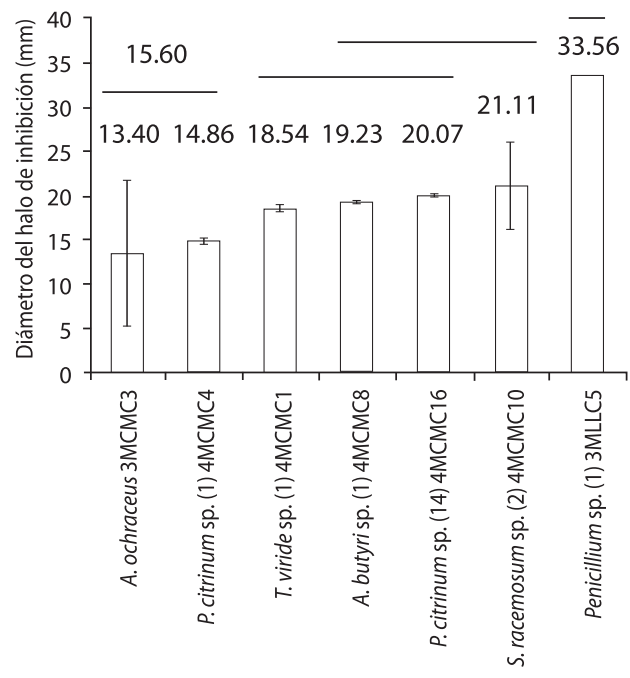

Hongos marinos

Fig. 1. Eficiencia de extractos crudos de hongos marinos sobre $P$. aeruginosa ATCC 9027; los datos fueron normalizados por la expresión $\log _{10}$, ANOVA I $(\alpha=0.01$; $\mathrm{F}=31.49)$; Duncan $(\alpha=0.01)$.

Fig. 1. Efficiency of the raw extract of marine fungi on Pseudomonas aeruginosa ATCC 9027; the data were normalized by the expression $\log _{10}$, ANOVA I $(\alpha=0.01$; $F=31.49)$; Duncan $(\alpha=0.01)$.

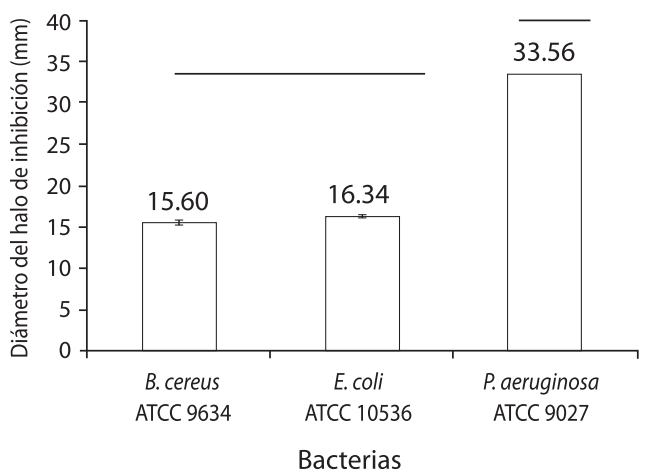

Fig. 2. Eficiencia del extracto crudo de Penicillium sp. (1) 3MLLC5 sobre bacterias ATCC; los datos fueron normalizados por la expresión $\mathrm{e}^{\mathrm{x}}$; ANOVA I $(\alpha=0.01 ; \mathrm{F}=511.19)$, Duncan $(\alpha=0.01)$.

Fig. 2. Efficiency of the raw extract of Penicillium sp. (1) 3MLLC5 on ATCC bacteria; the data were normalized by the expression $\mathrm{e}^{\mathrm{x}}$; ANOVA I $(\alpha=0.01 ; \mathrm{F}=511.19)$; Duncan $(\alpha=0.01)$. no se detectó inhibición en una cepa de $V$. parahaemolyticus; en ambos trabajos de investigación el extracto y/o metabolito obtenido de los hongos marinos ensayados manifestaron ser de espectro limitado. No obstante, en el presente estudio A. ochraceus 3MCMC3 y P. citrinum (14) 4MCMC16 inhibieron el $100 \%$ y $80 \%$ de los gérmenes reveladores respectivamente, los cuales exhibieron un amplio espectro.

Puede inferirse que el amplio espectro detectado en $A$. ochraceus 3MCMC3 y $P$. citrinum (14) 4MCMC16, pudiera deberse en principio, a que el área de la Laguna de Tacarigua presenta un impacto antropogénico elevado, favoreciendo el ingreso de grupos de bacterias coliformes a los microambientes de donde se aislaron los hongos marinos; unido a este hecho, al aumento de nutrientes y contaminantes, probablemente estimulen el desarrollo de cualidades degradativas y transformadoras por parte de las poblaciones microbianas (Atlas y Bartha 2002, Castillo y Malaver 2003); estos hechos posiblemente propicien la producción de metabolitos secundarios destinados al control de las poblaciones microbianas alóctonas por parte de las comunidades microbianas preestablecidas (De Freitas y Fredrickson 1978, Fredrickson y Stephanopoulos 1981). También la halogenación de los metabolitos secundarios en el medio marino genera variabilidad en el grupo de toxinas y enzimas provenientes de microorganismos marinos (Stander 2000). Se estima, que la combinación de todos estos factores pudiera incidir en la variabilidad de los metabolitos secundarios producidos por este grupo de hongos marinos lo cual probablemente sea la causa del amplio espectro obtenido.

En forma similar, el género Pseudomonas constituye parte de la flora bacteriana del suelo y de los fondos marinos (Atlas y Bartha 2002), por lo que puede darse el hecho de que algunas poblaciones de hongos allí establecidas produzcan metabolitos secundarios destinados al control de poblaciones perteneciente a este género bacteriano, lo cual coincide con los resultados obtenidos en el presente estudio, ya que $P$. aeruginosa 9027 , resultó ser inhibida por todos los extractos de hongos marinos ensayados. 
Los extractos obtenidos de A. ochraceus 3MCMC3 y $P$. citrinum (1) 4MCMC4, se muestran como fuentes potenciales para el aislamiento de metabolitos secundarios con propiedades antibacterianas de amplio espectro, y el extracto de Penicillium sp. (1) 3MLLC5 con propiedades inhibidoras de cepas pertenecientes a $P$. aeruginosa.

Basados en los resultados obtenidos se recomienda aislar sustancias bioactivas con propiedades antibacterianas, provenientes de los extractos seleccionados y determinar sus estructuras moleculares.

\section{RESUMEN}

La actividad antibacteriana de extractos de hongos marinos se evaluó mediante una prueba de eficiencia, para tal fin los hongos fueron inoculados en medio Agar Extracto Malta (EMA) con $50 \%$ de agua de mar y período de crecimiento de 60 días. Se obtuvieron extractos con una solución de acetona/cloroformo/etanol (3:2:1). La fase orgánica se trató a presión reducida con rotaevaporador (baño de María incorporado a $40^{\circ} \mathrm{C}$ ). Con los extractos se realizaron antibiogramas por triplicado. Se midieron los diámetros de los halos de inhibición y se les aplicó una prueba de eficiencia empleando un ANOVA I, y prueba a posteriori Duncan. Los extractos de Aspergillus ochraceus 3MCMC3 y Penicillium citrinum (14) 4MCMC16 presentan propiedades antibacterianas de amplio espectro, inhibiendo el $100 \%$ y $80 \%$ de los gérmenes reveladores, con énfasis hacia la inhibición de Pseudomonas aeruginosa 9027, en especial el extracto de Penicillium (1) 3MLLC5 presentó la mayor eficiencia sobre esta cepa; también se encontró que P. aeruginosa 9027 y Escherichia coli 10536 fueron ser los gérmenes reveladores más sensibles. Los hongos marinos seleccionados se perfilan como una fuente de metabolitos secundarios antibacterianos.

Palabras clave: actividad antibacteriana, hongos marinos, raíces de mangle, Rhizophora mangle, Laguna de Tacarigua, Venezuela.

\section{REFERENCIAS}

Atlas, R. \& R. Bartha. 2002. Ecología microbiana y microbiología ambiental. Addison Wesley, Pearson Educación, Madrid, España.
Bauer, A., W. Kirby, I. Sherris \& M. Turk. 1966. Antibiotic susceptibility testing by standardized single disk method. Am. J. Clin. Pathol. 45: 493-496.

Christophersen, C., O. Crescente, J. Frisvad, L. Gram, J. Nielsen, P. Nielsen \& L. Rahbaek. 1999. Antibacterial activity of marine-derived fungi. Mycopathología 143:135-138.

De Freitas, M. \& G. Fredrickson. 1978. Inhibition as a factor in the maintenance of the diversity of microbial ecosystems. J.G. Microbiol. 106: 307-320.

Fredrickson, A. \& G. Stephanopoulos. 1981. Microbial competition. Science 213: 972-979.

Hoeller, U., G. Koenig, \& A. Wright. 1999. Three new metabolites from marine-derived fungi of the genera Coniothyrium and Microsphaeropsis. J. Nat. Prod. 62: 114-118.

Kelecom, A. 2002. Secondary metabolites from marine microorganisms. An. Acad. Bras. Cienc. 74: 151170 .

Lin, Y., X. Wu, Z. Deng, J. Wang, S. Zhou, L. Vrijmoed \& E. Gareth Jonesc. 2002. The metabolites of the mangrove fungus Verruculina enalia No.2606 from a salt lake in the Bahamas. Phytochemistry 59: 469-471.

Nielsen, J., P. Nielsen \& J. Frisvad. 1999. Fungal depside, guisinol, from a marine derived strain of Emericella unguis. Phytochemistry 50:263-265.

Shigemori, H., Y. Kasai, K. Komatsu, M. Tsuda, Y. Mikami \& J. Kobayashi. 2004. Sporiolides A and B, new cytotoxic twelve-membered macrolides from a marine-derived fungus Cladosporium species. Mar. Drugs. 2:164-169.

Sperry, S., G. Samuels \& P. Crew. 1998. Vertinoid poliketides from the saltwater culture of the fungus Tricoderma longibrachiatum separated from a Haliclona marine sponge. J. Org. Chemist. 63: 10 011-10 014.

Stander, M., P. Steyn, A. Lübben, A. Miljkovic, P. Mantle \& G. Marais. 2000. Influence of halogen salts on the production of the ochratoxins by Aspergillus ochraceus Wilh. J. Agric. Food Chem. 48: 1865.

Zar, J. 1984. Biostadistical Analysis. Prentice Hall, Nueva Jersey, EEUU. 
